\title{
Radio data and synchrotron emission in consistent cosmic ray models
}

\author{
Torsten Bringmann \\ II. Institute for Theoretical Physics, University of Hamburg, Luruper Chausse 149, \\ D-22761 Hamburg, Germany \\ E-mail: torsten.bringmann@desy.de
}

\section{Fiorenza Donato}

Dipartimento di Fisica Teorica, Università di Torino, Istituto Nazionale di Fisica Nucleare, via P. Giuria 1, I-10125 Torino, Italy

E-mail: donato@to.infn.it

\author{
Roberto A. Lineros \\ IFIC, CSIC-Universidad de Valencia, Ed. Institutos, Apdo. Correos 22085, E-46071 \\ Valencia, Spain \& MultiDark fellow \\ E-mail: rlineros@ific.uv.es
}

\begin{abstract}
It is well established that phenomenological two-zone diffusion models of the galactic halo can very well reproduce cosmic-ray nuclear data and the observed antiproton flux. Here, we consider lepton propagation in such models and compute the expected galactic population of electrons, as well as the diffuse synchrotron emission that results from their interaction with galactic magnetic fields. We find models in agreement not only with cosmic ray data but also with radio surveys at essentially all frequencies. Requiring such a globally consistent description strongly disfavors very large ( $L \gtrsim 15 \mathrm{kpc})$ and, even stronger, small ( $L \lesssim 1 \mathrm{kpc}$ ) effective diffusive halo sizes. This has profound implications for, e.g., indirect dark matter searches.
\end{abstract}

\section{Introduction}

A wealth of observational data strongly suggests that diffusion governs the propagation of galactic cosmic rays (CRs) [1]. Any model for the underlying physical processes requires a basic assumption about the geometry of the region the CRs sweep. In diffusive models, the Galaxy is represented by a thin disk sandwiched by a thick magnetic diffusive halo with cylindrical symmetry. Given the intrinsic limitations to any such model, as well as present-day CR data, a considerable uncertainty in the propagation parameters is generally unavoidable.

A realistic 3D modeling turns to extensive computer codes, such as Galprop [2, 3] or Dragon [4], aiming at a detailed description of sources, interstellar matter distribution, 
magnetic field structure and diffusion phenomena. While reaching a high degree of accuracy - particularly needed for the gamma-ray component - such an approach is computationally expensive and does not always make it straight-forward to extract physical answers for the underlying processes and input parameters. An effective 2D approach, on the other hand, benefits from analytical solutions to the spatial diffusion equation [5, 6, 7], which allows fast computations and thus efficient scans of the parameter space [8]. Despite a small number of free parameters, it can consistently describe both nuclear CRs [5], CR antiproton [9] as well as electron and positron data [10, 11].

Here, we investigate whether even radio data can be understood in this 2D framework. We start by inferring the galactic electron distribution from an interpretation of large-scale radio survey data as synchrotron radiation and then compare this to the expectation in our propagation model. We find that radio observations are indeed consistent with models that correctly describe CR data, providing a remarkable hint that our effective propagation model is not too far from a real picture of galactic phenomena, at least on kpc scales. Furthermore, we show that our procedure can be used, in principle, as a new method to constrain propagation models which is complementary to using the boron over carbon $(\mathrm{B} / \mathrm{C})$ ratio [5] or radioactive isotopes ratios (i.e. $\left.{ }^{10} \mathrm{Be} /{ }^{9} \mathrm{Be}\right)[3$, 7, 8].

The structure of this article is as follows. We start by reviewing, in Section 2 , how relativistic electrons produce synchrotron radiation when propagating through the galaxy. In Section 3, we compute the galactic electron distribution in our diffusion model and compare the expected synchrotron radiation in Section 4 to radio surveys at various frequencies, demonstrating that synchrotron radiation indeed provides a very promising means of both inferring properties of the interstellar electron distribution and to provide constraints on the adopted diffusion model. After a discussion of possible biases in our analysis in Section 5, we present our conclusions in Section 6 ,

\section{Synchrotron radiation}

Relativistic electron $\ddagger$ emit synchrotron radiation while propagating through the galactic magnetic field [12]. For electrons with energy $E$ and a magnetic field of strength $B$, the emission power per unit frequency is given by

$$
\frac{d w}{d \nu}=\frac{\sqrt{3} e^{3} B}{m_{e} c^{2}} \frac{2}{\pi} \int_{0}^{\pi / 2} d \theta \sin \theta F\left(\frac{\nu}{\nu_{c} \sin \theta}\right),
$$

where $\nu_{c}=3 e B E^{2} /\left(4 \pi m_{e}^{3} c^{5}\right), F(x)=x \int_{x}^{\infty} d \zeta K_{5 / 3}(\zeta)$ and $K_{5 / 3}$ is a modified Bessel function; we take the average over the angle $\theta$ between the electron momentum and $\vec{B}$ because we assume an isotropic electron distribution (note also that, in general, not only the regular but also the turbulent component of the galactic magnetic field contributes to the total signal - which further motivates this average).

$\ddagger$ Unless explicitly stated otherwise, we will in the following use the term electron to denote both electrons and positrons. 
For a given electron density $n_{e}(E, \mathbf{x})$, the expected intensity in synchrotron radiation is thus given by

$$
I_{\nu}=\frac{1}{\Delta \Omega} \int_{\Delta \Omega} d \Omega \int d \ell J_{\nu}(\mathbf{x}) e^{-\int_{0}^{\ell} d \ell^{\prime} \alpha_{\nu}\left(\mathbf{x}^{\prime}\right)}
$$

where $J_{\nu}=\int \frac{d n_{e}}{d E} \frac{d w}{d \nu} d E$ is the emissivity, $\alpha_{\nu}$ the inverse of the absorption length and the integrations are taken along the line of sight towards the observed direction in the sky, averaged over an angular region $\Delta \Omega$. Since radio emission is often associated with thermal phenomena, the intensity is traditionally stated in terms of temperature: with the Rayleigh-Jeans law in mind, $I_{\nu}=2 \nu^{2} k_{B} T / c^{2}$, one can define the brightness temperature as

$$
T_{b} \equiv I_{\nu} c^{2} /\left(2 \nu^{2} k_{B}\right) .
$$

The galactic magnetic field has an average strength of $\mathcal{O}(\mu \mathrm{G})$ [13]. While its detailed structure in reality can be expected to be rather sophisticated [14, we will here adopt an effective approach and treat it to be spatially constant within the diffusion zone as we will only be interested in an average, large-scale description of radio data above the galactic plane. In fact, this simplifying assumption seems necessary in order to be consistent with the homogeneous diffusion coefficient that enters as a basic ingredient to our propagation model. We verified that adopting instead a magnetic field falling off exponentially away from the disk - in principle also consistent with the geometry of our diffusion model - does not change significantly our predictions for the synchrotron flux (integrated along the line of sight) with respect to our choice of a constant magnetic

field that abruptly vanishes at the border of the diffusion zone. This small difference can readily be understood in terms of the propagation scale length, which is $\sim 300 \mathrm{pc}$ for electrons with energies $\sim 3 \mathrm{GeV}$ : most of the electrons will simply remain relatively close to the galactic disk, where the magnetic field distributions are similar, thereby reducing the differences in the integrated synchrotron emission.

For our analysis, we take into account absorption of synchrotron photons by both thermal electrons and ions from a possible hot gas component in the disk [15. Synchrotron self-absorption is another potential effect, but for $B \sim \mu \mathrm{G}$ it becomes numerically important only for frequencies below $1 \mathrm{MHz}$ [16] where it is however dominated by free-free absorption. Finally, let us mention that for an electron distribution following a power law, $d n_{e} / d E \propto E^{-\gamma}$, Eqs. (1.3) tell us that also the synchrotron intensity, in the case of negligible absorption, follows a power law, $T_{b} \propto \nu^{-\alpha}$, with a spectral index

$$
\alpha=(\gamma+3) / 2 .
$$

\section{Galactic electron population}

The transport parameters of the two-zone diffusion model introduced in Ref. [5] are determined from the $\mathrm{B} / \mathrm{C}$ analysis and correspond to the size of the diffusive halo of the Galaxy $L$, the normalization of the diffusion coefficient $K_{0}$ and its slope $\delta$ (defined by 
Radio data and synchrotron emission in consistent cosmic ray models

\begin{tabular}{l||c|c|c||c|c}
\multicolumn{1}{c||}{ Model } & \multicolumn{2}{c||}{ prop. parameters } & \multicolumn{2}{c}{ radio data $\left(\chi^{2} /\right.$ d.o.f. $)$} \\
& $L[\mathrm{kpc}]$ & $K_{0}\left[\frac{\mathrm{kpc}^{2}}{\mathrm{Myr}}\right]$ & $\delta$ & $408 \mathrm{MHz}$ & $1.42 \mathrm{GHz}$ \\
\hline $\min$ & 1 & 0.0016 & 0.85 & $11.6(6.8)$ & $11.9(6.3)$ \\
\hline $\operatorname{med}$ & 4 & 0.0112 & 0.70 & $4.9(2.0)$ & $4.9(2.0)$ \\
\hline $\max$ & 15 & 0.0765 & 0.46 & $10.8(4.8)$ & $8.9(3.9)$
\end{tabular}

Table 1. Benchmark models compatible with B/C data [17. Both 'min' and 'max' are clearly disfavored by radio data towards the galactic anti-center (averaged over $10^{\circ}$ $\left(15^{\circ}\right)$ and excluding the disk).

$K=K_{0} \beta R^{\delta}$, where $R=p / q$ is the rigidity), with a rather strong degeneracy between the allowed parameters - in particular between $K_{0}$ and $L$. Diffusive reacceleration and convection, while crucial for the nuclei analysis, have been shown to be only mildly relevant for lepton fluxes [10]. Indeed, the two processes shape $\mathrm{GeV}$ electron fluxes in the opposite direction, so that their combined effect is rather small; for that reason, and for the sake of simplicity, we do not take them into account here. In this seminal analysis, we will for simplicity mainly refer to the three benchmark propagation models shown in Table 1, among all models compatible with B/C data, these were shown to give the minimal, medium and maximal flux in antiprotons, respectively, that is expected from dark matter annihilations in the galactic halo [17].

High energy electrons are produced in galactic accelerators such as supernova remnants (SNRs) or pulsars (primary electrons), as well as in hadronic interactions of galactic protons and helium nuclei with the interstellar medium (secondary electrons). We calculate the primary $e^{-}$flux from SNRs following Ref. [11 and the subdominant secondary $e^{ \pm}$component as described in Ref. [10], using a cylindrical gas density distribution in the disk as given in Ref. [18. For the energy losses, we take into account inverse Compton scattering off the interstellar radiation field, as well as synchrotron, bremsstrahlung and ionization losses in the interstellar medium [8].

\section{Comparison to radio data}

Starting from the 1960s, several groups have performed large-area radio surveys in the frequency range from about $1 \mathrm{MHz}$ to $100 \mathrm{GHz}$; for an extensive list, we refer the reader to Ref. [19]. In this reference, the surveys at 0.010 [20], 0.022 [21], 0.045 [22], 0.408 [23], 1.42 [24], 2.326 GHZ [25], as well as WMAP foregrounds at 23, 33, 41, 61 and $94 \mathrm{GHz}$ [26], were transformed to galactic coordinates and pixelized. In Fig. 1, we show the north and south pole at these frequencies, with the isotropic CMB component removed and averaged over a circular region with $10^{\circ}$ in diameter.

At these frequencies and latitudes, the dominant source of the radio signals should be synchrotron radiation (except for the excess seen in the $94 \mathrm{GHz}$ band which is probably due to spinning dust [27] and which we will not consider in the following). Freefree emission in general becomes important at frequencies $\nu \gtrsim 1 \mathrm{GHz}$, but is physically 


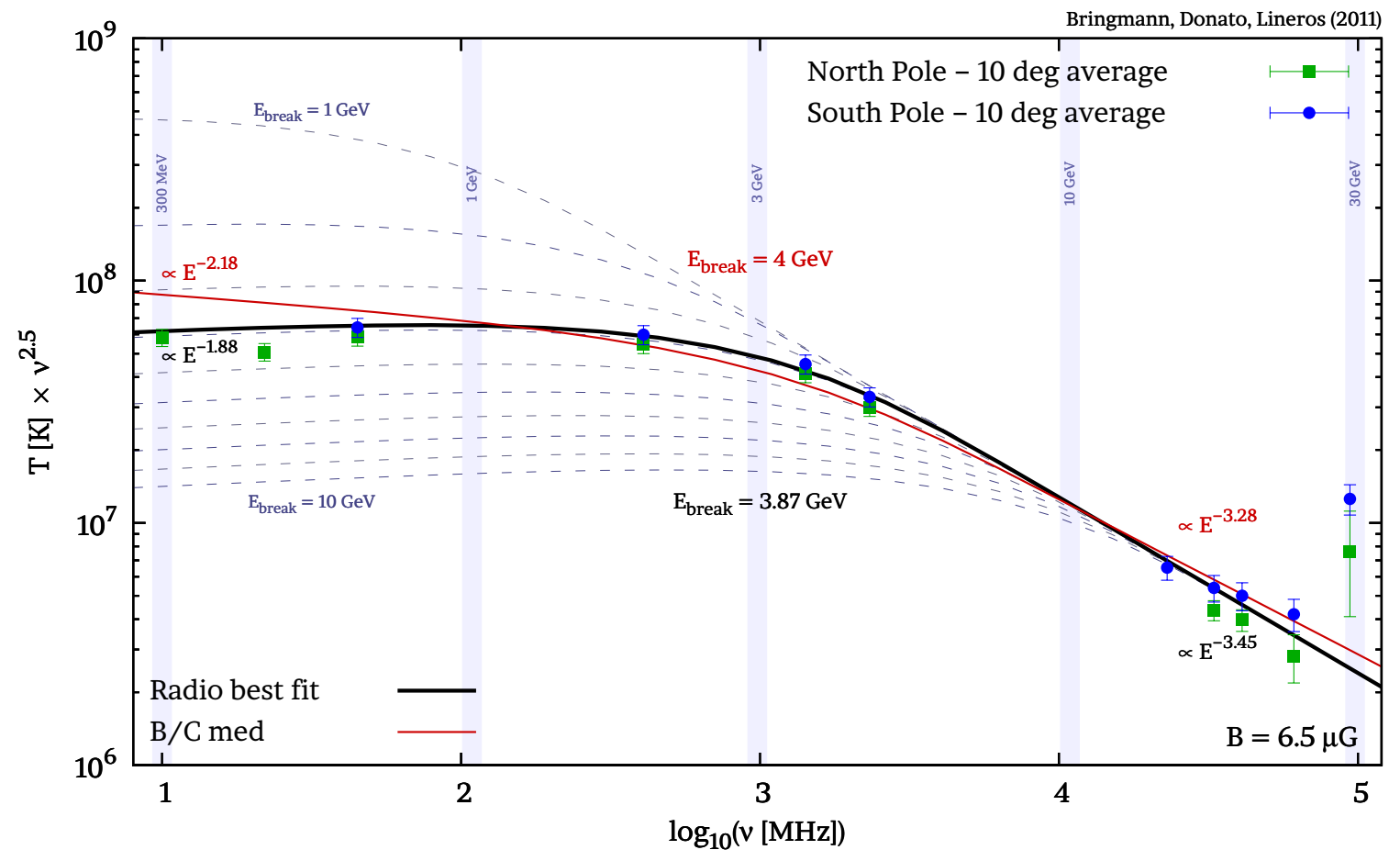

Figure 1. Observed radio fluxes vs. frequency. Data points are generated using the GSM software [19] which exactly reproduces the observational data at $10 \mathrm{MHz}$ [20], $22 \mathrm{MHz}$ [21], $45 \mathrm{MHz}$ [22], $408 \mathrm{MHz}$ [23], $1.42 \mathrm{GHz}$ [24, $2.326 \mathrm{GHz}$ [25], as well as WMAP foregrounds at 23, 33, 41, 61 and $94 \mathrm{GHz}[26$. The thick solid line represents the best fit for synchrotron radiation from an electron population (after propagation) with $d n_{e} / d E \propto E^{-\gamma_{1}}\left(d n_{e} / d E \propto E^{-\gamma_{2}}\right)$ for $E<E_{\mathrm{br}}\left(E>E_{\mathrm{br}}\right)$; the dashed lines indicate the effect of changing the best-fit value $E_{\mathrm{br}}=3.9 \mathrm{GeV}$. The thin solid line shows a fit with a break in the energy losses for the 'med' model of Tab. 1 see text for further details.

subdominant when looking away from the galactic disk: adopting values for the thermal electron temperature and distribution as given in Ref. [28], we estimate the highest freefree contribution (at $61 \mathrm{GHz}$ ) to be less than $20 \%$ in a cone towards the poles; given the error bars shown in Fig. 1, this does not have a significant effect on our analysis. From the data, one can clearly distinguish two regimes with a different power-law behavior in frequency - which directly translates to the necessity of a break in the spectral index of the propagated galactic electron population. If the propagated electrons are simply modeled with a broken power law, the best-fit values for $d n_{e} / d E$ are a break in the spectral index $\Delta \gamma=1.57_{-0.25}^{+0.2}$ at $E_{\mathrm{br}}=3.87_{-1.17}^{+1.43} \mathrm{GeV}$, with $\gamma_{2}=3.45_{-0.15}^{+0.15}$ above $E_{\mathrm{br}}$ (note that the sharp break in the electrons gets smoothed because we use the full expression (1) for the synchrotron power rather than the often adopted monochromatic approximation). This result is fully consistent with, e.g., that of Strong et al. [36], see their Fig. 5, and displayed as a thick, black solid line. Here, we chose a fiducial value of $B=6.5 \mu \mathrm{G}$; a different value would simply change the location of $E_{\mathrm{br}}$ and the (arbitrary) overall normalization (by a factor $\propto B^{2}$ ), but not the functional dependence on $\nu$ (we 


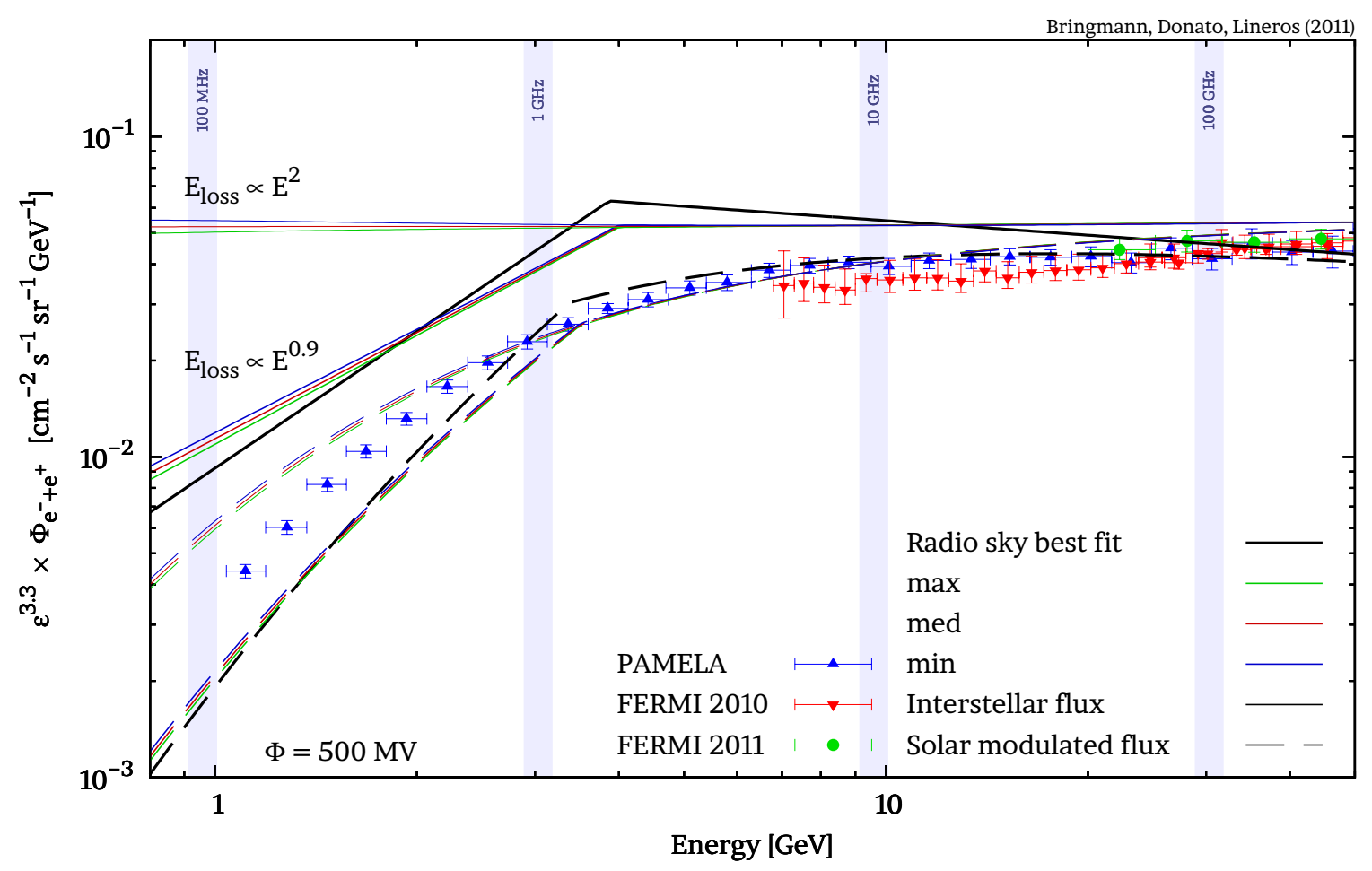

Figure 2. Measured spectrum in CR electrons ( $e^{-}$for the case of PAMELA) below $50 \mathrm{GeV}$ [29, 30, 31] and predictions according to Refs. [10, 11], assuming a spectral break in the energy losses. This spectrum is consistent with the radio data shown in Fig. 1 (vertical bands indicate the corresponding frequency of synchrotron radiation).

take the opportunity to remind the reader that $B=6.5 \mu \mathrm{G}$ is an effective value which takes into account both the average regular and turbulent component of the galactic magnetic field).

Such an electron distribution is actually also in rather good agreement with the observed CR electron fluxes (note that only electrons well below $100 \mathrm{GeV}$ are relevant to our discussion because the spectrum at higher energies is likely to be dominated by local sources). In Fig. 2, we show the Pamela $e^{-}$data [29] together with the $e=e^{+}+e^{-}$data taken by Fermi-LAT [30]. Very recently, the Fermi-LAT Collaboration has performed a separation between observed positrons and electrons above $20 \mathrm{GeV}$ using the Earth magnetic field as a spectrometer [31], confirming their previous results on the total lepton fluxes.

Along with the data, we plot in Fig. 2 theoretical predictions for the interstellar and solar modulated fluxes. Black lines correspond to the radio best fit (see Fig. 1), where electrons are naively shaped by two power laws with break of $\Delta \gamma=1.57$. We also show the resulting flux at Earth after propagation according to Refs. [10, 11] and for the propagation models listed in Table 1; here, we included for comparison a break of 1.1 in the energy loss term, making the hypothesis that losses effectively follow $E^{0.9}$ below $E_{\text {break }}$ (for the 'med' model, this is in addition shown as a red solid line in Fig. 1). In order to obtain the curves shown in Fig. 2, we choose the normalization and spectral 
index $\gamma_{\text {inj }}$ of the primary $e^{-}$spectrum in such a way as to fit the total (primary and secondary) $e^{-}$spectrum to the low-energy PAMELA data (we checked that adding the Fermi electron data, given their energy range and error bars, would not significantly affect this normalization). We stress that our normalization is consistent with Fig. 1, i.e. it reproduces both the the radio data (with $B=6.5 \mu \mathrm{G}$ as stated above) and, roughly, the low-energy lepton data [29].

For energy losses that do not significantly change during the typical path an electron propagates, the spectral index of the expected electron flux is approimately given by [11]

$$
\gamma \approx \gamma_{\mathrm{inj}}+\frac{1}{2}(\beta+\delta-1)
$$

where $\gamma_{\text {inj }}$ is the spectral index of the injected electrons (before propagation) and the energy loss term is assumed to scale like $b \equiv-\partial_{t} E \propto E^{\beta}$. This relation is not more valid, however, if electrons experience different energy loss regimes while wandering through the Galaxy, depending for example on the time spent in the halo (which is gas free) or in the disk. If energy losses are dominated by a term specific to the local environment where the electron spends most of its time, $b \propto E_{\text {loc }}^{\beta}$, one can derive from the general expressions given in Ref. [11] that the expected relation for the observed spectrum instead becomes

$$
\gamma \approx \gamma_{\mathrm{inj}}+\frac{1}{2}(-\beta+\delta-1)+\beta_{\mathrm{loc}}
$$

which reduces to Eq. (5) for $\beta_{\mathrm{loc}}=\beta$. We note therefore that the break illustrated in Figs. 1 and 2, taking into account the viable scatter in $\Delta \gamma$, might simply reflect the fact that at lower energies the scattering on thermal ions and electrons $\left(b_{\text {loss }} \propto E\right)$ becomes more important than inverse Compton losses $\left(b_{\text {loss }} \propto E^{2}\right)$. This interpretation is also consistent with the location of the break that is determined by the relative strength of these processes: taking into account uncertainties in the radiation, magnetic field and gas densities, we expect the transition to occur in the range $1 \mathrm{GeV} \lesssim E_{\text {break }} \lesssim 10 \mathrm{GeV}$. In fact, as also shown in Figs. 1 and 2 , a corresponding value of $\Delta \beta_{\text {loc }}=1.1$ (which we choose slightly larger than 1 in order to capture possible effects of reacceleration) does show a very reasonable agreement with both radio and lepton data.

Let us now turn to the angular shape of the radio signals, which we show in Fig. 3 for the case of the Haslam 23. map at $408 \mathrm{MHz}$. In the same figure, we indicate the expectation for the models of Tab. 1, including the full variation of the parameters $K_{0}$ and $\delta$ within the range compatible with the $\mathrm{B} / \mathrm{C}$ analysis performed in Ref. [5]. One can clearly see that the size of the diffusive halo has a rather strong impact on the angular shape of the resulting synchrotron signal. Demanding consistency with B/C data, small halo sizes $L \sim 1 \mathrm{kpc}$ are essentially excluded, but also large values $L \gtrsim 15 \mathrm{kpc}$ show some tension with radio data. Note that the magnetic field normalization does not affect the angular shape of the synchrotron emission; here, it was chosen such as to be consistent with Figs. 1 and 2. 


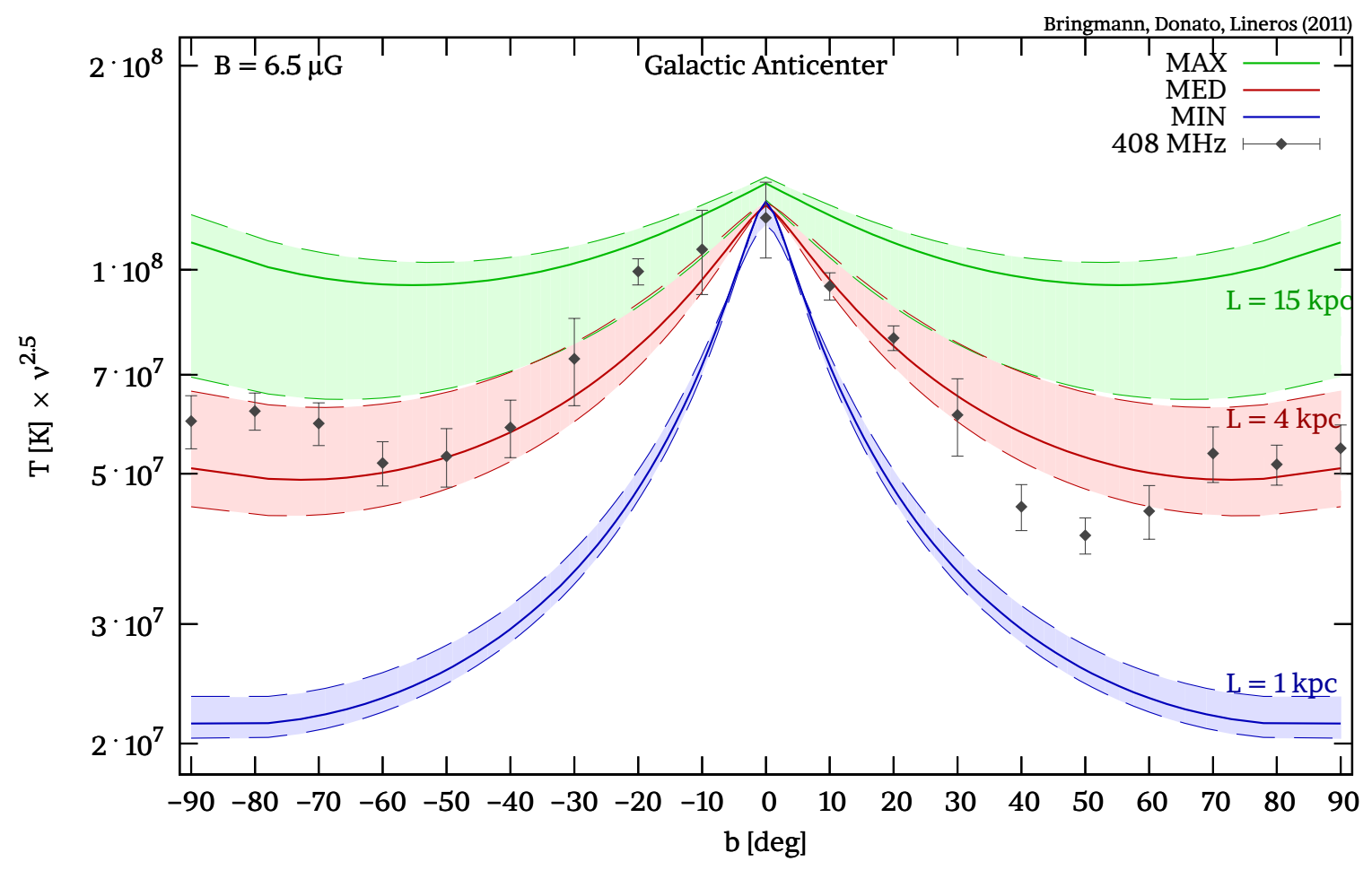

Figure 3. Brightness temperature versus latitude at $408 \mathrm{MHz}$. Solid lines correspond to the propagation models given in Table 1 . The shaded areas are obtained by varying $K_{0}$ and $\delta$, at fixed $L$, over the full range compatible with $\mathrm{B} / \mathrm{C}$ data.

In order to demonstrate the constraining power of the angular shape of radio data for diffusion models, we next treated the overall normalization of the synchrotron signal (aka the magnetic field) as a free parameter to minimize $\chi^{2}$ for the $\mathrm{min} / \mathrm{med} / \mathrm{max}$ models. The result is shown in Tab. 1 for the $408 \mathrm{MHz}$ and $1.42 \mathrm{GHz}$ maps; as anticipated, it is very difficult to reconcile radio data with the max and, even more so, the min model. Similar conclusions apply to all surveys with $\nu<408 \mathrm{MHz}$, while for $\nu>1.42 \mathrm{GHz}$ the data generally start to reproduce the expected synchrotron pattern worse, even for the med model; this is likely due to molecular clouds and pulsars (and/or SNRs), mostly in the southern hemisphere, that produce bremsstrahlung emission. We note that even the overall fit quality, for the min model, is quite good for angular averages of at least $15^{\circ}$; on smaller scales, on the other hand, we can obviously not expect our effective model to reproduce the detailed features that are visible in the radio sky. While these results already indicate the power of the method and thus warrant a more detailed

analysis of the propagation parameter space and the magnetic field structure [32], this is beyond the scope of the present work.

\section{Discussion}

Let us now discuss which assumptions in the analysis presented above are affected by uncertainties that might bias our conclusions. We have explicitly verified that the 
absorption of synchrotron radiation by thermal electrons is negligible for $\nu \gtrsim 10 \mathrm{MHz}$ and insensitive to the thermal electron temperature (for $4000 \mathrm{~K}<T_{e}<8000 \mathrm{~K}$ ) below this frequency; for very low values $\left(T_{e} \lesssim 3000 \mathrm{~K}\right)$ absorption modifies the spectral shape in a way strongly disfavored by the data. Weak uncertainties are derived from the ensuing bremsstrahlung radiation at high frequencies, whose contribution emerges around the $\mathrm{GHz}$ and gets stronger with lower $T_{e}$.

We checked that a break in the diffusion coefficient does not induce relevant changes in the frequency spectrum, in contrast to the break in the (total) propagated spectrum discussed above; we found that a break in the source spectrum of primary electrons alone, on the other hand, would result in an overproduction of synchrotron radiation from secondary electrons at frequencies $\nu \lesssim 100 \mathrm{MHz}$. We also verified that modifying $K_{0}$ or $L$ merely shifts the spectrum w.r.t. $\nu$ by changing its normalization; as a function of $\nu$, the three benchmark models of Tab. 1, e.g., simply scale up the spectrum by a factor of 5 when going from min to max. A degeneracy in $B, K_{0}$ and $L$ (marginally also $\delta$ ) becomes manifest even when looking at the radio spectrum as a function of the latitude. It is only when we turn to physical (i.e. B/C compatible) values of the propagation parameters that the degeneracy is partially broken in the spectrum w.r.t. the latitude, see Fig. 3 .

\section{Conclusions and Outlook}

The connection between synchrotron radiation and radio data provides a very interesting means of inferring properties of the interstellar galactic electron population at low energies [33]. We have shown that the radio sky from $\mathrm{MHz}$ to $\mathrm{GHz}$, when averaged over large scales, can be understood in terms of synchrotron emission of diffused galactic $e^{ \pm}$predicted in a model consistent with many other cosmic observables, including CR nuclei (from protons to iron), radioactive isotopes and antiprotons. This is a rather new result and strong evidence that we are not too far from having a global picture of the phenomena occurring in the galaxy.

We also found first indications that such a description breaks some of the degeneracies encountered when constraining the properties of the diffusive halo with nuclear CR data. A more detailed analysis of the allowed space of propagation parameters will be treated in a forthcoming publication [32]. However, let us stress that we have already presented preliminary evidence for $1 \mathrm{kpc} \lesssim L \lesssim 15 \mathrm{kpc}$. We note that in particular the $\bar{p}$ flux from dark matter annihilations in the galactic halo is mostly sensitive to the volume probed by the magnetic diffusion zone; a lower bound on $L$ will thus have important implications for indirect dark matter searches.

\section{Acknowledgments}

We thank A. Strong and M. Regis for valuable discussions and acknowledge use of the HealPix software [34]. We also would like to thank the anonymous referee for 
useful comments that helped to improve this article. TB was supported by the German Research Foundation (DFG) through Emmy Noether grant BR 3954/1-1. FD and RL thank the LEXI initiative and the theoretical astroparticle group at the university of Hamburg for financial support and hospitality. RL was supported by the EC contract UNILHC PITN-GA-2009-237920, by the Spanish grants FPA2008-00319, FPA201122975, MultiDark CSD2009-00064 (MICINN), and PROMETEO/2009/091 (Generalitat Valenciana).

Note added. In the final stage of preparing this manuscript, we became aware of Refs. [35, 36] also investigating the complementary information from CR and synchrotron data on galactic electrons (with, however, a somewhat different focus compared to ours).

\section{References}

[1] A. W. Strong, I. V. Moskalenko, V. S. Ptuskin, Ann. Rev. Nucl. Part. Sci. 57, 285 (2007) astro$\mathrm{ph} / 0701517$.

[2] A. W. Strong, I. V. Moskalenko, Astrophys. J. 509, 212 (1998) astro-ph/9807150; I. V. Moskalenko, A. W. Strong, Astrophys. J. 493, 694 (1998) astro-ph/9710124; R. Trotta, G. Johannesson, I. V. Moskalenko, T. A. Porter, R. R. de Austri, A. W. Strong, Astrophys. J. 729, 106 (2011) arXiv:1011.0037.

[3] I. V. Moskalenko, A. W. Strong, S. G. Mashnik, J. F. Ormes, Astrophys. J. 586, 1050 (2003) astro-ph/0210480.

[4] C. Evoli, D. Gaggero, D. Grasso, L. Maccione, JCAP 0810, 018 (2008) arXiv:0807.4730; G. Di Bernardo, C. Evoli, D. Gaggero, D. Grasso, L. Maccione, Astropart. Phys. 34, 274 (2010) arXiv:0909.4548.

[5] D. Maurin, F. Donato, R. Taillet, P. Salati, Astrophys. J. 555, 585 (2001) astro-ph/0101231.

[6] D. Maurin, R. Taillet, F. Donato, Astron. Astrophys. 394, 1039 (2002) astro-ph/0206286].

[7] F. Donato, D. Maurin, R. Taillet, Astron. Astrophys. 381, 539 (2002) astro-ph/0108079.

[8] A. Putze, L. Derome, D. Maurin, Astron. Astrophys. 516, A66 (2010) arXiv:1001.0551.

[9] F. Donato, D. Maurin, P. Salati, A. Barrau, G. Boudoul, R. Taillet, Astrophys. J. 563, 172 (2001) astro-ph/0103150; T. Bringmann, P. Salati, Phys. Rev. D75, 083006 (2007) astro-ph/0612514; F. Donato, D. Maurin, P. Brun, T. Delahaye, P. Salati, Phys. Rev. Lett. 102, 071301 (2009) arXiv:0810.5292.

[10] T. Delahaye, F. Donato, N. Fornengo, J. Lavalle, R. Lineros, P. Salati, R. Taillet, Astron. Astrophys. 501, 821 (2009) arXiv:0809.5268.

[11] T. Delahaye, J. Lavalle, R. Lineros, F. Donato, N. Fornengo, Astron. Astrophys. 524, A51 (2010) arXiv:1002.1910.

[12] For details see, e.g., V. L. Ginzburg, S. I. Syrovatskii, Ann. Rev. Astron. Astrophys. 3, 297 (1965).

[13] J. L. Han, IAU Symp. 259, 455 (2009) arXiv:0901.1165.

[14] X. H. Sun, W. Reich, A. Waelkens, T. Enslin, Astron. Astrophys. 477, 573 (2009) arXiv:0711.1572 ; M. S. Pshirkov, P. G. Tinyakov, P. P. Kronberg, K. J. Newton-McGee, Astrophys. J. 738, 192 (2011) arXiv:1103.0814.

[15] J. D. Peterson, W. R. Webber, Astrophys. J. 575, 217 (2002).

[16] G. B. Rybicki, A. P. Lightman, Radiative Processes in Astrophysics, Wiley-VCH (2004).

[17] F. Donato, N. Fornengo, D. Maurin, P. Salati, Phys. Rev. D69, 063501 (2004) astro-ph/0306207.

[18] A. Misiriotis, E. M. Xilouris, J. Papamastorakis, P. Boumis, C. D. Goudis, Astron. Astrophys. 459, 113 (2006) astro-ph/0607638.

[19] A. de Oliveira-Costa, M. Tegmark, B. M. Gaensler, J. Jonas, T. L. Landecker, P. Reich, arXiv:0802.1525 [astro-ph].

[20] J. L. Caswell, MNRAS 177, 601 (1976). 
[21] R. S. Roger, C. H. Costain, T. L. Landecker, C. M. Swerdlyk, Astron. Astrophys. Supplement 137, 7 (1999) astro-ph/9902213.

[22] A. E. Guzman, J. May, H. Alvarez, K. Maeda, Astron. Astrophys. 525, A138 (2011) arXiv:1011.4298.

[23] C. G. T. Haslam, C. J. Salter, H. Stoffel, W. E. Wilson, Astron. Astrophys. 47, 1 (1982).

[24] P. Reich and W. Reich, Astron. Astrophys. Suppl. Ser. 63, 205 (1986).

[25] J. L. Jonas, E. E. Baart, G. D. Nicolson, MNRAS 297, 977 (1998).

[26] A. de Oliveira-Costa, M. Tegmark, Phys. Rev. D74, 023005 (2006) astro-ph/0603369].

[27] A. de Oliveira-Costa, M. Tegmark, R. D. Davies, C. M. Gutierrez, A. N. Lasenby, R. Rebolo, R. A. Watson, Astrophys. J. 606, L89 (2004) arXiv:astro-ph/0312039.

[28] M. I. R. Alves, R. D. Davis, C. Dickinson, M. Calabretta, R. Davis and L. Staveley-Smith, arXiv:1108.6268.

[29] O. Adriani et al. [PAMELA Collaboration], Phys. Rev. Lett. 106, 201101 (2011) arXiv:1103.2880].

[30] M. Ackermann et al. [Fermi-LAT Collaboration], Phys. Rev. D82, 092004 (2010) arXiv:1008.3999.

[31] M. Ackermann et al. [Fermi-LAT Collaboration], arXiv:1109.0521

$[32]$ T. Bringmann, F. Donato and R. Lineros, work in progress.

[33] for earlier work see, e.g., M. L. Goldstein, R. Ramaty, L. A. Fisk, Phys. Rev. Lett. 24, 1193 (1970); A. C. Cummings, E. C. Stone, R. E. Vogt, ICRC 1, 335 (1973); J. M. Rockstroh, W. R. Webber, Astrophys. J. 224, 677 (1978); A. E. E. Rogers, J. D. Bowman, Astron. J. 136, 641 (2008) arXiv:0806.2868]; A. Tartari, M. Zannoni, M. Gervasi, G. Boella, G. Sironi, Astrophys. J. 688, 32 (2008) arXiv:0806.4306]; E. Orlando, A. W. Strong, I. V. Moskalenko, T. A. Porter, G. Johannesson, S. W. Digel, arXiv:0907.0553 A. E. Guzman, J. May, H. Alvarez, K. Maeda, Astron. Astrophys. 525, A138 (2011) arXiv:1011.4298 and references therein.

[34] K. M. Gorski, E. Hivon, A. J. Banday, B. D. Wandelt, F. K. Hansen, M. Reinecke, M. Bartelmann, Astrophys. J. 622, 759 (2005).

[35] T. R. Jaffe, A. J. Banday, J. P. Leahy, S. Leach, A. W. Strong, MNRAS 416, 1152 (2011) arXiv:1105.5885.

[36] A. W. Strong, E. Orlando and T. R. Jaffe, Astron. Astrophys. 534, A54 (2011) arXiv:1108.4822. 